\title{
A partial matching theory of the mirror effect in immediate probed recognition
}

\author{
DAVID J. MURRAY, JASON BURHOP, STEPHEN CENTA, NIKIL CHANDE, KIRSTEN OINONEN, \\ TERRY THOMAS, TREENA WILKIE, and BAHAR FARAHMAND \\ Queen's University, Kingston, Ontario, Canada
}

\begin{abstract}
A set of experiments on immediate probed recognition of digit triples is reported in which the variables were list length (five, six, seven, or eight triples), the probability that a probe was old (.33, .5 , or $.67)$, and whether the digit triples were presented with an auditory component or articulatory suppression. Previous work had suggested that the false alarm (FA) rate in this paradigm was lower when auditory information was available than when it was not; this observation had led to the development of the partial matching theory of immediate probed recognition, according to which FAs could arise not only as a result of unlucky guesses but also when new probes shared a first digit in common with a partially retained target triple. It was argued that partial memory representations were less likely following auditory presentation than following articulatory suppression. Partial matching theory is contrasted with the rational response theory, according to which all FAs are unlucky guesses; partial matching theory gave a better account of the present experimental data than did rational response theory. However, a logical relationship between the two theories was suggested, a consequence of which was that rational response theory could be modified to include partial matching in such a way as to account for mirror effects, not only in unusually difficult immediate probed recognition tasks, but also in the more commonly studied mixed test list paradigm involving words of high or low frequency.
\end{abstract}

These six experiments were first described in honours theses by J.B., S.C., N.C., K.O., T.T., and T.W. They were supervised by D.J.M. in the academic year 1994-95. Diane E. Birch assisted substantially in the development of the computer program, originally designed by Lorraine Farrell, that controlled the presentation of the targets and probes and summarized the subjects' responses in terms of hit rates, false alarm rates, and other summary statistics. N.C. adapted Birch's computer program so that the probability of an old probe could be independently manipulated and was also responsible for suggesting how the degree of partial matching between new probes and targets might best be calculated. T.T. initiated the move to try to estimate false alarm rates on the basis of partial matches between retained elements and elements of new probes. S.C. corrected an earlier error made in the development of rational response theory. Sylvia Hains carried out all the analyses of variance and calculations of $d_{L}, P_{r}, C_{L}, B_{r}$, and standard errors using the Queen's University mainframe computer. The personal computers used in these experiments were also provided by Queen's University. Maureen Freedman and Rachel Murray typed the manuscript, B.F. aided substantially in drafting the manuscript and in reanalyzing data collected earlier, Rob Ellis taught us how to use spreadsheets provided by Microsoft Excel, and Monica Hurt did the illustrations. Some of the expenses of manuscript preparation were borne by a research grant from the Social Sciences and Humanities Research Council of Canada to D.J.M. He is grateful to B. B. Murdock, Jr., William E. Hockley, and Andrew Heathcote for helpful advice during the early stages of the development of the rational response theory. He also wishes to express his gratitude to R. L. Greene and Ian Neath for their helpful and encouraging reviews of earlier submissions concerning this research; in particular, he is extremely grateful to Peter Dixon for the care with which he constructively criticized more than one draft of the final version of the paper, gradually shaping it with his comments into its present form. Correspondence should be addressed to D. J. Murray, Department of Psychology, Queen's University, Kingston, ON, K7L 3N6 Canada (email: murrayd@pavlov.psyc.queensu.ca).

-Accepted by previous associate editor Peter Dixon
In the recent literature on human recognition memory, it has frequently been found that with two experimental conditions $\mathrm{A}$ and $\mathrm{B}$, if $\mathrm{A}$ is associated with a higher hit rate than is $\mathrm{B}, \mathrm{A}$ will also be associated with a lower false alarm (FA) rate than is B. In the most common example of experimental conditions that have yielded this "mirror effect" (Glanzer \& Adams, 1985), Condition A consists of a list of low-frequency words and Condition B consists of a list of high-frequency words. Typically, a list that contains equal numbers of low- and high-frequency words is presented along with some kind of instructions-for example, to decide whether each word is indeed a word (the list will also include some nonwords or very rare words), or to say each word aloud, or to answer a question about each word; we shall call this the study list or target list. Following a retention interval (typically minutes, hours, or longer), a test list or probe list consisting of all or some of the target words intermingled with an equal number of new words (i.e., words that were not presented in the target list) is presented and subjects must judge whether each of these test words is "old" or "new." The new words include both low- and high-frequency words. The usual finding is that low-frequency words have higher hit rates than do high-frequency words, but also that low-frequency words have lower FA rates than do highfrequency words. Glanzer and Adams (1985) gave many examples of this mirror effect from the literature available at that time and noted that word frequency was not the only variable to yield the mirror effect; other variables that did so included word-imageability, concreteness, meaningful- 
ness (familiarity), emotionality, number of dictionary meanings, pronounceability, and associative frequency. Mirror effects have also been obtained when the words consisted of nouns or non-nouns (Greene, 1996; Hockley, 1994); pictorial materials have also yielded mirror effects (see Glanzer \& Adams, 1985, Table 5; Vokey \& Read, 1992).

A general name for this kind of experiment, which involves long-term memory and in which the materials are varied, is the mixed test list paradigm, so named because the test list includes a mixture of the two kinds of material. However, mirror effects can also be obtained in experiments that focus on short-term memory (now often called "working memory") in which the variables that are manipulated do not concern the materials so much as the sensory modality of presentation of the target items, probe items, or both. For example, Wickelgren and Norman (1966) presented lists of two to seven spoken digit triples (e.g., 218,639, etc.) and, following each list, presented either one of the triples again (with a probability of .67) or a new triple that had not been in the list. The subject had to say whether this "probe" triple was "old" or "new." The obtained hit rates as list length increased from two to seven triples were $1.0, .971, .878, .847, .733$, and .685 , respectively; the corresponding FA rates were $.003, .013$, $.077, .082, .071$, and .136 , respectively. A mirror effect is clearly visible, and list length is the variable that is apparently associated with the different levels of difficulty that yield different hit rates.

More recently Murray et al. (in press) have demonstrated that if a target list of eight digit triples is presented at a rate of $2 \mathrm{sec}$ per triple and is followed immediately by a single probe triple that is either old (with a probability of $.5)$ or new with respect to the target list, target lists that involved an auditory component were associated with higher hit rates than were target lists that had been seen silently with no auditory component; but the lists with auditory components also yielded lower FA rates than did the lists seen silently. Here the variable that seems to be determining the mirror effect is the presence or absence of auditory information heard directly by the subject. Other studies that have investigated immediate probed recognition for digit triples include those by Murray, Rowan, and Smith (1988), who explored the effects of articulatory suppression on performance, and Murray, Birch, Chase, Eyolfson, and Simms (1991), who explored the effects of varying the intensity level of the auditory components of the target presentation; these investigations will be discussed later.

There are at least four explanations of the mirror effect available in the literature concerning long-term recognition tasks for two kinds of material as opposed to the single material (digit triples) investigated by Wickelgren and Norman (1966) and by Murray and his colleagues. First, Glanzer and Adams (1985, pp. 18-19) put forward (and dismissed) a theory according to which subjects guess in a particular way when a probe is unfamiliar; we shall here call this the rational response theory and de- velop it further below as well as report experiments deliberately devised to test its validity. Second, Brown, Lewis, and Monk (1977) suggested that when a probe (or a lure in a forced-choice task) appeared that was unfamiliar, subjects would ask whether that probe or lure itself was so "memorable" (recallable) that, if the subject could not recognize it as an "old" item in the context of the experiment, it must necessarily be responded to as "new" because otherwise the subject would have recognized it. Despite its psychological plausibility, this theory received little support from experiments designed to evaluate it by Hintzman, Caulton, and Curran (1994); and Heit and Jones (1997) have argued that, although hit rates in recognition tasks are positively related to the accuracy with which the targets can be recalled, FA rates do not appear to vary with recall accuracy.

Third, there is the theory of Glanzer and Adams (1985) themselves, according to which the mirror effect is the outcome of an inference process in the subject's mind during which he/she decides whether a probe should be judged "old" or "new" on the basis of a decision as to whether the probe came from a sample drawn from a low-familiarity range of possible probes (new probes) or a high-familiarity range (old probes). The decision is based on the value the probe has with respect to a scale that measures the relative frequencies with which a new probe and an old probe were associated with any given value of subjective familiarity, this measure being based on the ratio of the two frequencies for that value. The scale was called the "log likelihood ratio" scale. Glanzer, Adams, Iverson, and Kim (1993) have argued that a computer simulation of obtained mirror effects, mainly found in experiments in which two kinds of material were involved, such as high- versus lowfrequency words, yielded predictions of FA rates that correlated significantly with obtained FA rates when the computer calculated those values of certain parameters that maximized the probability of such a conformity. The model involved four distributions, a "signal" and a "signal-plus-noise" distribution concerning materials belonging to one type, A (say, low-frequency words), and a signal and a signal-plus-noise distribution for materials belonging to another type, B (say, high-frequency words). Arranged along an axis representing "familiarity," the mean of the type A "signal" distribution, representing the "easier" condition, lay to the right of the mean of the type B "signal" distribution. But the theory hypothesized that the means of the two signal-plus-noise distributions, corresponding to new probes of the two kinds of material, were arranged so that the mean representing new probes of type A (the easier condition) lay to the left of the mean representing new probes of type B. This hypothesis is unique to the theory of Glanzer and Adams and is necessary if the mirror effect is to be explained by that theory.

Glanzer and Adams deliberately avoided including data from experiments in which the test lists consisted of either high- or low-frequency words exclusively, a design used, for example, by McCormack and Swenson 
(1972). But Glanzer and Adams (1985, p. 12, note 9) explicitly singled out this study as perhaps excludable from their survey, even though McCormack and Swenson did obtain a mirror effect with their "pure test list" as well as with their "mixed test list" design. The reason for Glanzer and Adams's focus on experiments involving test lists containing both kinds of material as new probes was that the model of Glanzer and his colleagues assumes that, in old/new tasks, the setting of a single criterion by subjects determines the hit rates ("old" responses to old probes) and the FA rates ("old" responses to new probes) for both kinds of material.

Fourth, Shiffrin and Steyvers (1997) have criticized extant models of the mirror effect that depend on an adjustable single criterion because "they usually provide no reason why the subject should adjust the criterion in the required way [for different lists]" (p. 150). Shiffrin and Steyvers offered an alternative theory based on the storage of incomplete and error-prone copies ("images") about which the subject makes a decision according to rules based on Bayes' theorem. This theory predicts mirror effects based on list length but shares with the theory of Glanzer and his colleagues the idea that the decision process can be modeled in terms of a criterion setting within the context of the signal detection model. Their model, moreover, was also focused on studies of recognition memory for words of high or low frequency in which the test lists consisted of a large number of mixed old and new words of either kind of frequency.

But in the experiments reported by Wickelgren and Norman (1966) and by Murray and his colleagues, even though the same subjects provided hit and FA rate data within each of the experiments, in all cases there was only one probe after each list; that is, on all trials we had a "pure" one-probe test list. Nor did any target lists include different kinds of material or demand different kinds of modality or articulation within a list. Moreover, the number of targets in all those studies was eight triples at most; the variable manipulated was list length in Wickelgren and Norman's experiment and encoding modality in the experiments of Murray et al. (in press), not the kind of material. Finally, the use of long lists and retention intervals of minutes or hours, rather than seconds, in the mixed test list paradigm would ensure that subjects used secondary as well as primary memory in this paradigm.

In the present paper, we offer two theories designed to account for mirror effects in immediate probed recognition. One is the rational response theory mentioned above; the other is a theory that we have not encountered elsewhere, the partial matching theory. First we argue that the latter provides a good account of the patterns of hit and FA rates in immediate probed recognition, but also hypothesize that rational response theory may come into play when the task is so difficult as to require extensive guessing. We then sketch how a modified version of rational response theory that includes a component based on partial matching can provide at least an initial account of the mirror effect in the mixed test list paradigm. The modified rational response theory, like its unmodified simpler version and like partial matching theory, is distribution free, and one of the main aims of the paper is to argue that distribution-free theories of the mirror effect are feasible and present an alternative to theories based on the signal detection model.

\section{Rational Response Theory}

The rational response theory has been previously expressed in the following way. Glanzer and Adams (1985, pp. 18-19) argued that a mirror effect could be predicted on the basis of some versions of two-high-threshold theory. If we have two conditions in one of which the hit rate is higher than the other and in both of which the probability of guessing "old," $g$, is constant, then for both conditions

$$
H^{\prime}=H+(1-H)(g)=g+(1-g) H
$$

and

$$
F A^{\prime}=(1-H)(g)=g-g H .
$$

Here $H^{\prime}$ is the predictcd hit rate, $H$ is the actual hit rate based on genuine memory matches, and $(1-H)(g)$ is the proportion of lucky hits. $F A^{\prime}$ is the predicted $F A$ rate. Note that if $g=.5, H^{\prime}$ and $F A^{\prime}$ sum to 1 ; but it is only true that $H^{\prime}$ and $F A^{\prime}=1$ when $g=.5$. A mirror effect is revealed because as $H^{\prime}$ values increase, the corresponding $F A^{\prime}$ values decrease; this is demonstrated by the final arrangement of terms in Equations 1 and 2, respectively. Note also that this argument completely ignores FAs arising from partial matching between new probes and retained targets, the topic of partial matching theory.

In this version of the theory, the term $g$ is often presumed to be given by the probability of an old probe, to be notated as $b$ in the text (or as $p$ [old probe] in the tables and figures), which, in the experiments by Murray and his colleagues, was always .5. In fact, Greene (1996) has argued that in the mixed test list design of the kind studied by Glanzer and his colleagues, even though an experimenter may not tell subjects that $b=.5$, subjects will generally assume this and apportion their guesses so that roughly half of all probes representing each of the two kinds of material will be judged "old" and half "new." Greene has ingeniously demonstrated that if subjects are instructed not to guess, FA rates fall drastically, as would be expected on the argument just presented, and the traditional mirror effect found in the recognition of nouns as opposed to non-nouns that occurs in very long target lists almost vanishes.

However, it must be realized that $g$ does not necessarily have to correspond to $b$, the experimentally preset probability of an old probe. This probability is defined in a frequentist manner as follows:

$$
b=\text { number of old probes/total number of probes }
$$

in the experiment. If subjects choose to guess according to some strategy other than that indicated by Greene (1996), even when the value of $b$ set by the experimenter is maintained at $.5, g$ may take on another value. How- 
ever, in all cases, rational response theory predicts that a plot of $F A^{\prime}$ (on the abscissa) in relation to $H^{\prime}$ (on the ordinate) will be linear, falling from the point $(0,1)$, representing perfect performance, to the point $(g, g)$, representing chance performance.

\section{Partial Matching Theory}

In contrast with rational response theory, partial matching theory predicts that if $b$ is varied by the experimenter and told to the subject in advance, this will not necessarily change either the predicted hit rates or the predicted FA rates. The reason is that partial matching theory argues that the main determinants of FAs are (1) the degree to which memory representations of the stimulus elements are tightly grouped and (2) the number of items (e.g., digit triples) in the list. According to this theory, the probability of an FA is a function of the number of accidental matches between part of a new probe (e.g., its first digit) and the corresponding part of an incompletely retained target trace. This probability in turn will clearly increase with the number of target traces that, for moderately short lists, will increase with the length of the list. Hence, according to partial matching theory, the key variables determining FA rates as well as hit rates will be the length of the list and the degree to which the digit triples in the list are stored in their entirety (as opposed to being stored partially-for example, in terms of first digits only); Murray et al. (in press) have argued explicitly that the degree to which digit triples are stored in their entirety in memory depends on whether the lists involve auditory (and/or articulatory) components or not. The effect of the variable $b$ will be minor or nonexistent.

The theory uses straightforward probability theory to predict hit rates and corresponding FA rates given a simple overwriting model as provided by Neath and Nairne (1995), and the corresponding FA rates given the assumption that an FA can arise when the first digit of a new probe accidentally matches the first digit of a target triple that has not been retained in its entirety, but only partially, by way of its first digit.

Neath and Nairne (1995) postulated that if we take a given target item $T_{1}$ in short-term store (basically a store of modality-specific features) and let it be followed by a later item $T_{2}$ that shares features with $T_{1}, T_{1}$ will be "overwritten" and forgotten with a certain probability. We shall make the simplifying assumption that if a target triple $T_{2}$ accidentally shares its first digit with a target triple $T_{1}$ that has entered store earlier, $T_{1}$ will be overwritten by $T_{2}$ with a probability equaling 1 . If $T_{2}$ occurs immediately after $T_{1}$, and given the fact that there were nine possible first digits of the triples used in the following set of experiments (zero was excluded), the probability that the first digits of $T_{1}$ and $T_{2}$ would match would be 1/9 (i.e., .111). This kind of probability will be labeled $m$ from now on. The probability that they would not match would therefore be $(1-.111)$ - that is, .889 .

The probability that $T_{1}$ will share its first digit with one of the $i$ subsequent items, and therefore be overwrit- ten, will be $\left[1-(.889)^{i}\right]$. Thus the probability that it will be remembered, $H_{i}$, will be $1-\left[1-(.889)^{i}\right],(.889)^{i}$. More generally,

$$
H_{i}=[1-m]^{i} \text {. }
$$

Using a rationale similar to that used for deriving $H_{i}$, we can predict the FA rate, $F A^{*}$, given a hit rate, $H^{*}$, for a particular list. First, we must estimate $H^{*}$ from the various $H_{i}$ values associated with the $L$ serial positions of a list containing $L$ triples. $H^{*}$ is given by summing the various values of $H_{i}$ and dividing by list length $L$ :

$$
H^{*}=\frac{\sum_{i=0}^{L-1} H_{i}}{L} .
$$

To derive $F A^{*}$, we assume that a list with $L$ target triples leaves in the subject's memory a total of $x$ fully known triples. We also assume that if a new probe accidentally shares only its first digit with one of those $x$ triples, the subject will correctly say "new" because of his/her complete memory for the triple in question. But if the first digit of the new probe accidentally matches one of a number of triples themselves remembered only by their first digit, the subject will say "old," thereby giving an FA. The problem is to determine retrospectively what that number is. We have again made a simplifying assumptionnamely, that when auditory or articulatory encoding is difficult, subjects adopt a strategy of trying to encode just the first digit of most or all of the target triples, especially under conditions involving articulatory suppression. A parallel strategy, remembering only the first letters of words, has been reported by Logie, Della Salla, Laiacona, Chalmers, and Wynn (1996) in the context of the recall of words. With a maximum of eight such digits in these experiments, it is plausible that the number in question simply equals $(L-x)$; that is, the number of targets left after $x$ fully encoded triples have been discounted on the grounds that correct responses of "new" to new probes would always be yielded even if that probe and one or more of the $x$ fully known triples in question shared a common first digit.

The probability that a new probe would not share its first digit with one of the $(L-x)$ partially encoded triples is $[1-m]$ raised to the power $(L-x)$; hence the probability that it would share its first digit with that of one of the $(L-x)$ items, and thereby be responded to as "old," gives the predicted FA rate

$$
F A^{*}=1-[1-m]^{L-x} .
$$

In practice, $x$ has to be estimated. It can be given either by $\sum H_{i}$ from $i=0$ to $i=(L-1)$ or by $\left(H^{*}\right) L$. The latter is convenient if, from an obtained hit rate, we wish to predict the associated FA rate given a prior knowledge of list length and $m$.

It must be remarked at this point, however, that Equation 5 will be appropriate only under certain conditions. If $x$ is defined as the number of target triples available in 
their entirety at the time of the new probe, and $(L-x)$ is defined as the number of target triples only partially encoded (by their first digits), but still available in memory at the time of the probe, this definition would have to be reconciled with the earlier contention that at least some of the $(L-x)$ triples may have been overwritten and are therefore not available in memory.

Let us denote the number of overwritten (forgotten) target triples by $k$ and the number of partially encoded triples still in memory by $p$. Then, if $k=0$,

$$
(L-x)=p,
$$

and, if $p=0$,

$$
(L-x)=k .
$$

But in fact both $p$ and $k$ may be non-zero. This issue will be revisited in the General Discussion; for the moment, however, we shall continue to use Equation 5 because, given that Equation 5 is concerned with $F A^{*}$, a measure that necessarily involves new probes, the probability that a new probe will accidentally have the same first digit as that possessed by two target triples (the earlier of which will have been overwritten) is so small for list lengths below 8 that Equation 5 can continue to serve as a valid predictor of the approximate value of $F A^{*}$ in the experiment now to be described. To be more precise: For list lengths $5,6,7$, and 8 , the probabilities that a new probe will have the same first digit as that possessed by two target triples are $.0093, .0128, .01596$, and .0189 , respectively. But we may note in advance that it follows from this that we might expect that any discrepancies that exist between predicted and obtained values of $F A^{*}$ as prescribed by Equation 5 should increase with list length.

Another, quite separate, reason for believing that Equation 5 can serve as a predictor for relatively short lists is as follows. If a target digit triple $T_{1}$ has a first digit that has been overwritten by the identical first digit of a subsequent triple $T_{2}$, it is possible that the subject noticed at the time of $T_{2}$ that "something," namely $T_{1}$, was forgotten. If a new probe also has that same first digit, he/she will know that it is new with respect to $T_{2}$, but guess that it is old with respect to the something that was forgotten.

For these two reasons, we believe that the exponent $(L-x)$ will adequately predict the obtained FA rate in immediate probed recognition tasks with relatively low values of $L$; however, strictly speaking, the exponent should be $(L-x-k)$.

The first set of experiments to be reported attempted to discover which of the two theories above appeared best to fit the data when the experimenters systematically varied the probability of an old probe $(b)$, list length $(L)$, and whether or not auditory memory components of target items were available. The variable named $b$ had three values-namely, $.33, .5$, and .67 ; the variable named $L$ had four values-namely, $5,6,7$, and 8 ; and the variable named target modality had two values-lists either were heard or were viewed with concurrent articulatory suppression. The heard lists were perceived under one of three conditions: Subjects (1) heard the target items spoken by somebody else, (2) spoke them aloud themselves as they saw them, or (3) heard the lists spoken by somebody else at the same time as the subjects saw them. The probe was heard in the same way as the targets (thereby reducing unwanted effects of delays due to decoding the probe, and/or of retrieval errors based on a failure of encoding specificity). There were three variants of the viewing of lists under articulatory suppression: (1) subjects said "the ... the" repeatedly (Condition The), (2) said the alphabet (starting with a random letter) (Condition Alph), or (3) spelled aloud a seven-letter word (given to them at the start of the list) (Condition Spell); in each of these three conditions, the probe was presented visually without concurrent articulatory suppression. The aim of these experiments was to determine how far each of the three variables could be used to predict FA rates, given observed hit rates. According to rational response theory, $b$ should be an important determinant of hit rates and FA rates; according to partial matching theory, $m$ and $L$ should be the main predictors of both hit rates and FA rates, with the variable $b$ having little predictive value with respect to these measures.

\section{METHOD}

\section{General Design}

In each of the six experiments, we used the immediate probed recognition task involving digit triples that had been used by Murray et al. (1988), Murray et al. (1991), and Murray et al. (in press). The target digit triples were presented visually one at a time (except when target triples were purely heard; see below) in the center of the screen of a personal computer, followed by the word WAIT (accompanied by a sound beep) for half a second, followed by the probe digit triple, which stayed on the screen until the subject responded "old" or "new" by pressing a key (the procedure is described in more detail below). The computer program was now modified so that instead of presenting old probes with a probability of .5 only, old probes could also be presented with probabilities of .33 or .67 It should be noted that subjects were always told in advance what this probability was. Note also that the total number of lists with new probes in an experimental session involving $b=.5$ must be considerably increased when $b=.33$ because in the latter case there will be twice as many trials with new probes as with old probes. But the calculation of obtained hit rate for any serial position in any condition for any subject has to be based on a number of trials with old probes that has to be the same for each of these two values of $b$ Each of the earlier studies had investigated recognition for individual triples for lists that always comprised eight triples $(L=8)$, but in the experiments to be described the list length could equal 5,6, 7 , or 8 because list length is a variable that plays a major role in the predictions of partial matching theory. The third new variable to be investigated, the comparison of lists encoded with auditory components with lists viewed under articulatory suppression, was controlled by instructions to the subjects.

Table 1 shows the layout of the six experiments. In all cases, immediate probed recognition was required of digit triples presented at a rate of 1,500 msec per triple ( $750 \mathrm{msec}$ on, $750 \mathrm{msec}$ off). Each experiment was devoted to collecting data for a single value of $b$ and for either auditory or articulatory suppression conditions (not both). For the three experiments involving auditory input, subjects were divided into three groups: one involving passively heard targets and probes, one involving seen-and-voiced targets and probes, and the 
Table 1

Summary of Conditions for Six Experiments

\begin{tabular}{|c|c|c|c|}
\hline Experimenter & $p(\text { Old Probe })^{*}$ & List Lengths ${ }^{\dagger}$ & Encoding Conditions \\
\hline T. Wilkie & .33 & $5,6,7,8$ & Hear, See-plus-voice, See-plus-hear \\
\hline N. Chande & .5 & $5,6,7,8$ & Hear, See-plus-voice, See-plus-hear \\
\hline S. Centa & .67 & $5,6,7,8$ & Hear, See-plus-voice, See-plus-hear \\
\hline T. Thomas & .33 & $5,6,7,8$ & The, Alph, Spell \\
\hline K. Oinonen & .5 & $5,6,7,8$ & The, Alph, Spell \\
\hline J. Burhop & .67 & $5,6,7,8$ & The, Alph, Spell \\
\hline
\end{tabular}

other involving seen and passively heard targets and probes. For the three experiments involving articulatory suppression, subjects were divided into the three groups indicated earlier--the, alph, and spell.

\section{Procedure}

In each of the six experiments, each subject was randomly assigned to one of the three groups (devoted to a single encoding method). After some initial practice (to be described below), he/she carried out four blocks of trials, each block being devoted to a single list length; the order in which the blocks were carried out was counterbalanced within each subject group. The number of trials in each block varied with $b$ and list length $L$. For example, in Experiment 2 , where $b=.5$, the number of trials on a given list length for a single subject was $2 L$; that is, for $L=5$, there were five trials with old probes (each probe testing a different serial position) plus another five trials with new probes. This made a total of 10 trials required for list length 5; over the single experimental session in which each subject participated, the subject did a total of $10+12+$ $14+16=52$ trials, given that he/she was tested with an old probe once on each serial position on each of list lengths $5-8$. Before starting the session, all subjects in all experiments received some practice trials using list length 6 viewed under the appropriate value of $b$ in the auditory or the articulatory suppression condition. The number of practice trials varied with $b$; for $b=.33$, it was 18 ; for $b=.5$, it was 12 ; and for $b=.67$, it was 9 . The total number of test trials in each session, with each subject being tested with old probes once on every serial position for each list length, were 78,52 , and 39 trials, respectively, for $b=.33, .5$, and .67 . Although in each experiment the hit rate for any serial position was based on 36 trials, most of the following data on hit rates are based on collapsing the data across serial positions. For example, each point in Figure 2, below, is based on hit rates yielded by $180,216,252$, or 288 trials with old probes and on FA rates yielded by $210,252,294$, or 336 trials with new probes on list lengths $5,6,7$, and 8 respectively.

Under the condition involving seen-and-voiced targets and probes, subjects spoke each target digit triple aloud in a normal voice as it appeared in the center of the computer screen (e.g., pronouncing 218 as "two one eight"). The probe digit triple was spoken aloud in the same way; it appeared $.5 \mathrm{sec}$ after the final target triple, this short interval being filled by the sound of a beep and the appearance of the word waIT on the screen. It was thus clear to subjects when the list ended and the triple on the screen was a probe. No specific instructions about speed of responding were given. The probe appeared on the screen until the subject had pressed a key indicating that he/she thought it was "old" or "new"; the key that was used to represent "old" was to the left of the keyboard for half the subjects and to the right of the keyboard for the other half within this condition. After this response had been given, the subject gave a confidence rating by pressing a key on the number pad from 1 to $7(1=$ guess; $7=$ very confident ). Once this rating had been given, the word REST appeared on the screen for $2 \mathrm{sec}$, followed by the word READY for $1 \mathrm{sec}$, followed by the first target of the next trial. If subjects wished to rest for longer between trials, they were told simply to delay pressing the confidence rating key until they were ready to proceed.

The condition with purely heard targets and probes involved the same sequence of events as described for the previous condition except that the screen was hidden from the subject's view by a card held by the experimenter; the experimenter spoke each triple aloud while the subject listened to him/her.

The condition with seen and passively heard targets and probes involved the same sequence of events except that the screen was now exposed to the subject's view; at the same time the subject heard the experimenter speak aloud the same digit triple at which the subject was looking.

For the three experiments involving articulatory suppression, each subject was similarly assigned to one of three groups; each group carried out its task using a particular type of articulatory suppression. Under Condition The, subjects said "the" aloud in rhythm with the digit triples; for Condition Alph, subjects were given a random letter of the alphabet spoken by the experimenter just before the onset of each list, and during the presentation of the list, the subject spoke the alphabet aloud starting with that random letter and starting the alphabet from $A$ again if he/she happened to arrive at $Z$ before the end of the target list. For Condition Spell, a seven-letter word was spoken to the subjects by the experimenter just before the onset of each list and the subject spelled it aloud as fast as desired, starting the spelling again if he/she had finished the first spelling before the target list was finished. The words all fell within the frequency range of 120 to 1,000 per million in the tables of word frequency provided by Kučera and Frances (1967).

\section{Response Data}

Data were obtained on mean hit rates (for each serial position) and mean FA rates (which do not vary with serial position) averaged over all the appropriate trials for a given list length for a given encoding condition for a given subject. The mean obtained hit and FA rates were aiso transformed into $d^{\prime}$ values using the tables of Elliott (1964).

\section{Subjects}

Each experiment involved 36 subjects, 12 in each group. All subjects were students from Queen's University enrolled in an introductory psychology course; they participated in the experiments as part of the requirement for credit in the course. No subject was tested in more than one session within an experiment or in any other of the six experiments in this set; that is, each subject came to only one experimental session.

\section{RESULTS}

Table 2 shows mean hit rates and mean FA rates associated with each list length and encoding condition in each of the six experiments. The means are averaged across serial position. 
Table 2

Mean Hit and False Alarm (FA) Rates for the Six Experiments Concerned With Varying $p$ (Old Probe)

\begin{tabular}{|c|c|c|c|c|c|c|c|c|}
\hline \multirow[b]{3}{*}{ Experiment Type } & \multirow[b]{3}{*}{ Condition } & \multirow{3}{*}{$\begin{array}{c}\text { List } \\
\text { Length }\end{array}$} & \multicolumn{6}{|c|}{$p$ (old Probe) and Experimenter } \\
\hline & & & \multicolumn{2}{|c|}{.33 (Wilkie) } & \multicolumn{2}{|c|}{.5 (Chande) } & \multicolumn{2}{|c|}{.67 (Centa) } \\
\hline & & & Hits & $\overline{\text { FAs }}$ & Hits & FAs & Hits & FAs \\
\hline \multirow[t]{14}{*}{ Heard targets } & Hear & 5 & .80 & .08 & .78 & .18 & .79 & .17 \\
\hline & & 6 & .67 & .14 & .74 & .21 & .76 & .06 \\
\hline & & 7 & .70 & .14 & .70 & .25 & .69 & .19 \\
\hline & & 8 & .70 & .22 & .70 & .19 & .69 & .31 \\
\hline & See plus voice & 5 & .80 & .10 & .77 & .18 & .86 & .12 \\
\hline & & 6 & .76 & .20 & .71 & .20 & .71 & .19 \\
\hline & & 7 & .74 & .16 & .76 & .18 & .75 & .19 \\
\hline & & 8 & .67 & .21 & .74 & .28 & .67 & .29 \\
\hline & See plus hear & 5 & .83 & .11 & .77 & .05 & .78 & .10 \\
\hline & & 6 & .79 & .15 & .74 & .06 & .74 & .14 \\
\hline & & 7 & .76 & .21 & .75 & .12 & .77 & .16 \\
\hline & & 8 & .75 & .21 & .76 & .14 & .78 & .19 \\
\hline & & & \multicolumn{2}{|c|}{.33 (Thomas) } & \multicolumn{2}{|c|}{.5 (Oinonen) } & \multicolumn{2}{|c|}{67 (Burhop) } \\
\hline & & & Hits & FAs & Hits & FAs & Hits & FAs \\
\hline \multirow[t]{12}{*}{ Articulatory suppression } & The & 5 & .68 & .21 & .63 & .17 & .73 & .27 \\
\hline & & 6 & .69 & .33 & .67 & .15 & .65 & .31 \\
\hline & & 7 & .75 & .38 & .55 & .24 & .69 & .37 \\
\hline & & 8 & .67 & .30 & .56 & .22 & .63 & .38 \\
\hline & Alph & 5 & .80 & .22 & .70 & .28 & .63 & .30 \\
\hline & & 6 & .67 & .30 & .67 & .40 & .56 & .50 \\
\hline & & 7 & .66 & .33 & .71 & .35 & .66 & .30 \\
\hline & & 8 & .67 & .30 & .65 & .36 & .68 & .33 \\
\hline & Spell & 5 & .55 & .30 & .67 & .22 & .63 & .40 \\
\hline & & 6 & .68 & .23 & .61 & .36 & .60 & .25 \\
\hline & & 7 & .56 & .27 & .64 & .31 & .65 & .42 \\
\hline & & 8 & .66 & .30 & .61 & .32 & .60 & .54 \\
\hline
\end{tabular}

Note - These means were obtained by averaging over serial positions, more details of which are provided in Figures 1 and 3 .

(a)

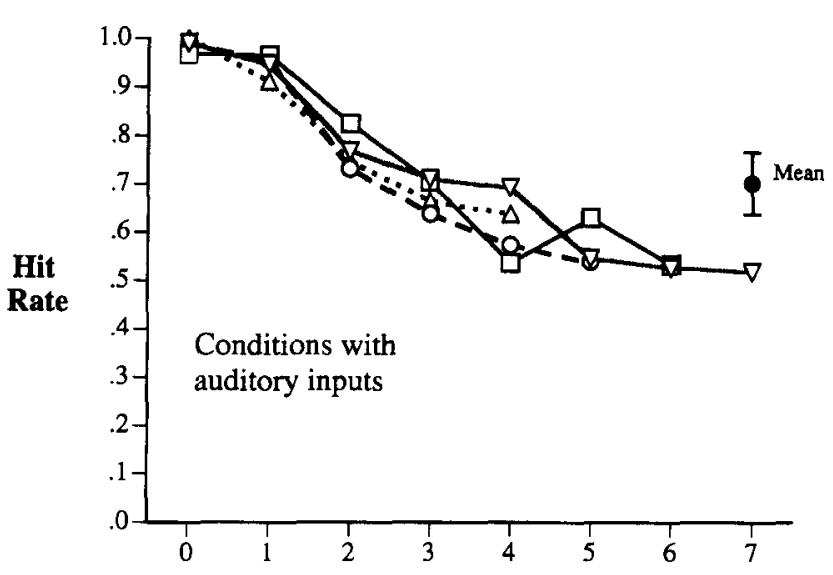

(b)

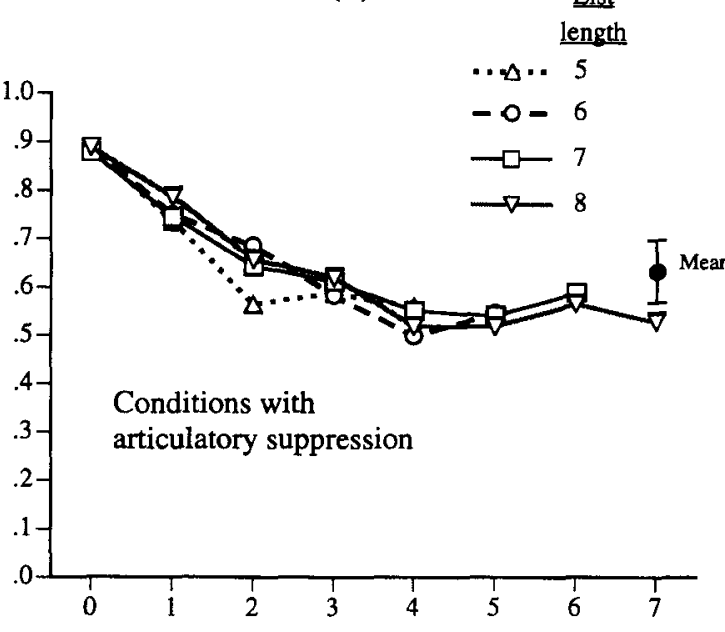

Number of items between target and probe (i)

Figure 1. (a) Mean hit rates as a function of the number of triples $(i)$ between target and probe for list lengths $5,6,7$, and 8 averaged across the three experiments in which subjects heard the targets in Experiment Set 1 . The data are also averaged over type of encoding condition in each experiment. (b) As for (a), but for the three experiments in Experiment Set 1 involving articulatory suppression. In both (a) and (b) the overall mean hit rate is shown separately; the error bar shows the mean value of the standard deviations for each serial position. These eight standard deviations are illustrated individually in Figure 3. 

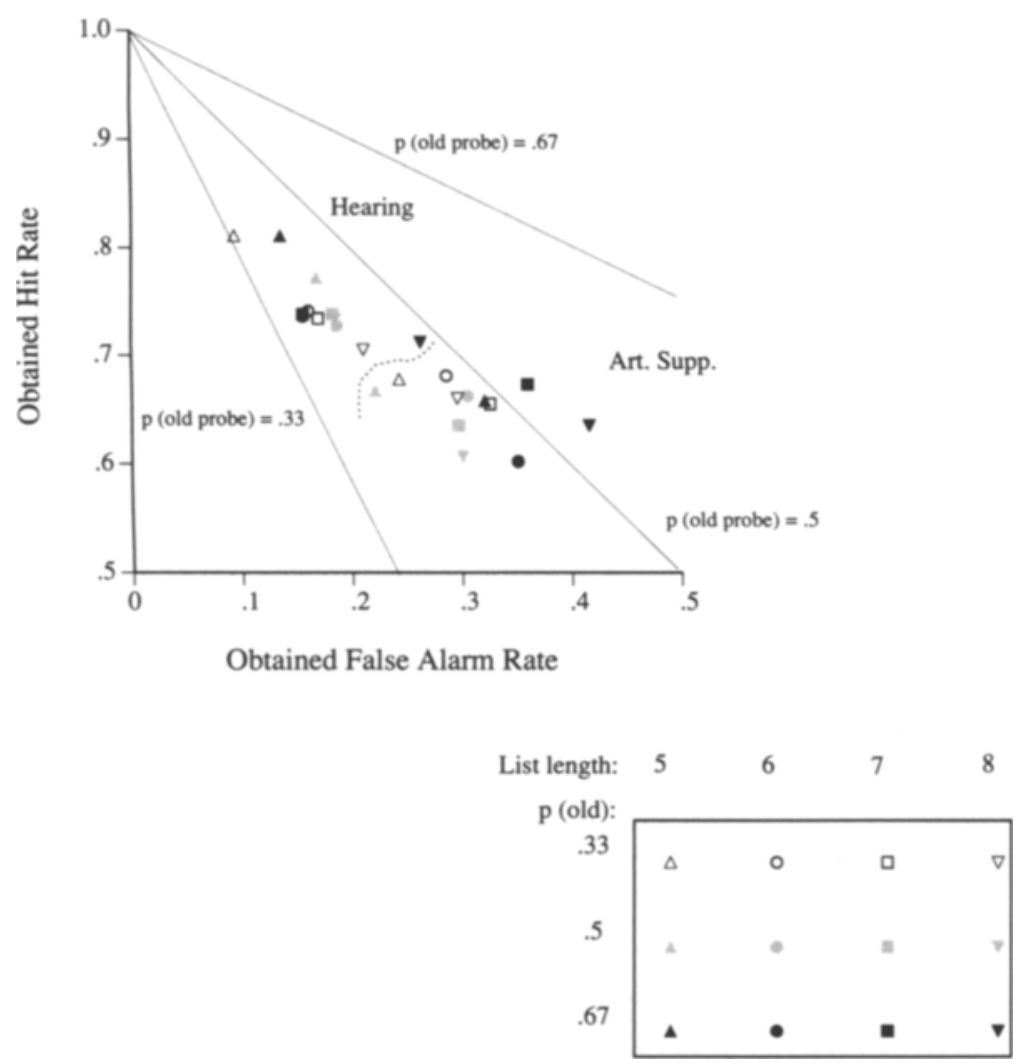

Figure 2. Obtained hit rates plotted as a function of obtained false alarm rates in the six experiments investigating hearing, articulatory suppression, list length, and $p$ (old probe). The straight lines show predictions made on the basis of rational response theory with $p$ (old probe) $=b=.33, .5$, or .67 . The data points to the left of the dotted line were obtained with hearing; those to the right involved articulatory suppression.

Analyses of variance (ANOVAs) were carried out separately on the hit rates for each serial position for each list length in each of the six experiments; it was found that the effect of serial position was significant at the .05 level in one analysis and at the .01 level in the remaining 23 analyses. More important, there was no significant serial position $X$ group effect in any of the 24 analyses and no main effect of group in 23 of the 24 ANOVAs. It appears, therefore, that for the purposes of the argument to follow, we are justified in collapsing the data for the three auditory conditions in each of the three experiments using auditory inputs and collapsing the data for the three conditions the, spell, and alph in each of the three experiments that used auditory suppression.

Figure 1 shows eight serial position curves, one for each list length, averaged over groups within experiments and values of $b$ across experiments. It can be seen that lists with auditory components showed higher levels of recency than did lists viewed under articulatory suppression, but it is striking how within each of these conditions the four serial position curves representing the four list lengths overlap over most of the range of serial positions. This fact can be used to justify a procedure of collapsing hit rates at any given serial position across the four list lengths for the hearing or the articulatory suppression conditions. We shall do so shortly, in the context of evaluating the applicability of partial matching theory to the present data.

If the mean hit rates and FA rates for each list length and each value of $p$ (old probe) for the hearing and articulatory suppression experiments are derived from Table 2 and plotted as in Figure 2, it is plain that the data points connected with $p$ (old probe) $=b=.33$ (open symbols) and those connected with $p$ (old probe) $=b=.67$ (black symbols) do not cluster around the respective straight lines for those two values of $b$ that are predicted by the rational response theory. Nor do they obviously group themselves along the line predicted by rational response theory for $b=.5$, as would be postulated if Greene's (1996) assumption extended even to cases where subjects were told that $b$ was .33 or .67 in advance. It would seem that subjects provided hit rates and FA rates that were determined by variables other than $b$.

The dotted line in Figure 3a shows the serial position curves for all the hearing conditions, with each point representing the mean hit rate for the items at a given serial 
- Predicted by PMT
$\ldots \ldots$ Obtained

(a)

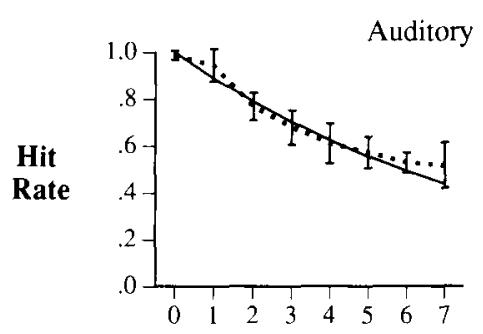

i (b)

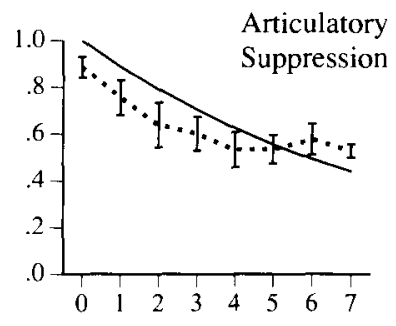

i (c)

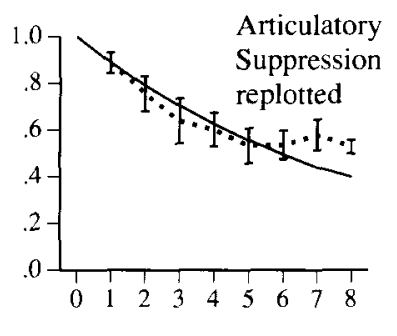

i

Figure 3. (a) The solid line shows predicted hit rate $H_{i}$ as a function of $i$ given partial matching theory with $m=$ .111. The dashed line shows the obtained hit rates averaged over all conditions involving heard targets in Experiment Set 1 . The error bar for each given point represents the standard deviation of the hit rates obtained for the 12 or fewer combinations of list length and $p$ (old probe) value associated with that point. (b) The solid line is the same as in (a), but the dashed line now represents obtained hit rates averaged over all experiments in Experiment Set 1 involving articulatory suppression. (c) Shows the same hit rates as in (b), but they are now plotted as if the last item were associated with extra interference due to articulatory suppression. So the obtained hit rate for the final item is now plotted against $i=1$ instead of $i=0$.

position and the mean being obtained by collapsing across groups, $b$ values, and list lengths-a procedure justified earlier. The solid line shows values of $H_{i}$ as predicted by the $i$ values shown on the abscissa. The Pearson correlation coefficient relating the eight obtained hit rates to the hit rates as predicted by $H_{i}$ was $r=.9815, d f=6, p<.00002$.

Figure $3 b$ shows the equivalent data for the articulatory suppression conditions. Clearly, the obtained hit rates lie below the curve predicted by $H_{i}$; the curve that best fits them is more conveniently discussed below.

Figure 4 shows exactly the same obtained data points as those of Figure 2, but the solid curve represents the expected values of $H^{*}$ given an associated value of $F A^{*}$ for the partial matching theory with $m=.111$. In view of the fact that the hit rates in the serial position curve for the articulatory suppression conditions in Figure $3 \mathrm{~b}$ were not well fitted by the predicted $H_{i}$ values that were summed in the process of deriving $H^{*}$ for each list length, it is surprising to see how well the solid line in Figure 4 seems to fit the obtained FA rates from the articulatory suppression experiments as well as the obtained FA rates from the experiments with heard targets.

That this impression can be supported by a statistical test can be shown by drawing a graph with predicted $F A^{*}$ rates on the abscissa and obtained FA rates (using the same scale) on the ordinate. If the former predicts the latter perfectly, the individual points will lay on a straight line with a slope of 1 , an intercept with the ordinate of 0 , and an associated Pearson correlation coefficient of +1 . In fact, this test yielded a line with a slope of 1.006, an intercept of -.0026 , and a Pearson $r$ of $.896(d f=22$, $p<.00001)$. However, it is also clear that the experiment yielding the FA rates that most departed from the partial matching theory function shown in Figure 4 was that of Burhop (spell, $b=.67, L=7$ and 8); we shall return to Burhop's data in the Discussion section.

A surprisingly simple explanation can account for the good fit of the solid curve in Figure 4 not only to the 12 obtained FA rates for the hearing conditions, but also to the 12 obtained FA rates for the articulatory suppression conditions. At least two different groups of authors have argued that the effect of articulatory suppression is not simply to prevent speech encoding of the targets, but to add material to memory--namely, the material enunciated aloud during articulatory suppression. Murray et al. (1988) presented an analysis of serial position curves for $d^{\prime}$ values obtained in immediate recognition of eight visually presented digit triples using various kinds of suppression tasks. They concluded, on the basis of Experiment 2 (by Andrea Rowan), that any kind of enunciatory activity that itself involved memory retrieval added material to store and that this had the effect of increasing the slope of the curve showing $d^{\prime}$ as a function of $i$. They argued that this increase in slope was compatible with the idea that, from a mathematical point of view, the particular values of the variable $i$ used in calculating the predicted slope of this curve under articulatory suppression should be increased from the corresponding values of $i$ used for an identical condition but from which articulatory suppression was absent. The latter are normally given as $i=0$ for the final item, and so on. Moreover, Neath and Nairne (1995) have argued that various phenomena connected with articulatory suppression can be explained if articulatory suppression adds a constant amount of material to the nonmodality (abstract) contents of long-term memory that are being processed in the context of that 

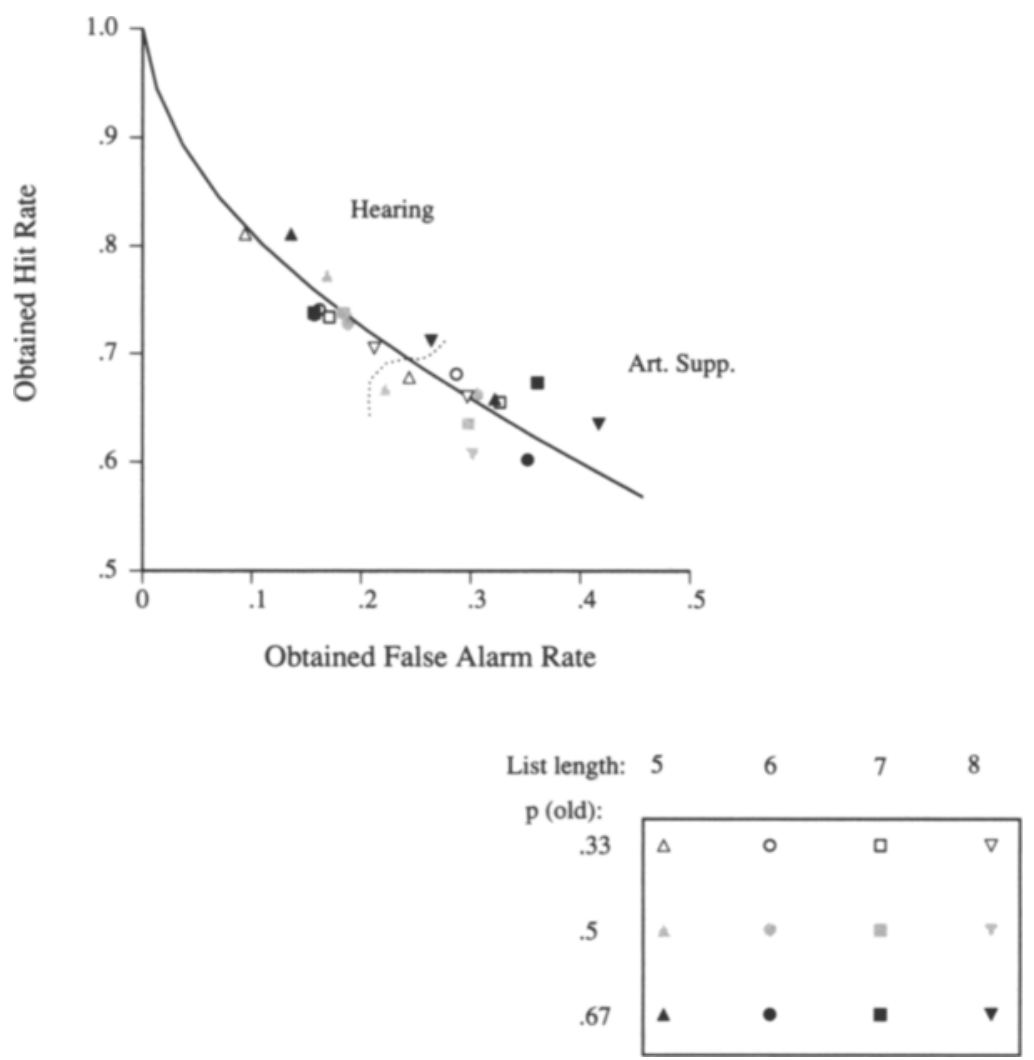

Figure 4. Mean obtained hit rates plotted as a function of mean obtained false alarm rates in the same manner as in Figure 2. The solid line shows the $H^{\star}$ and $F A^{*}$ values predicted by partial matching theory with $m=.111$. Each point on that curve represents a value of $i$.

particular experiment (ostensibly concerned with working memory).

If, therefore, we assume that, in the experiments whose obtained hit and FA rates are shown in Figure 4, the suppression activity added a constant amount into memory, the effect would be to lower $H^{*}$ and raise $F A^{*}$ to a point further rightward along the curve from the corresponding point for an otherwise identical hearing condition. Let this constant amount added to memory be the equivalent of one extra item. Figure $3 \mathrm{c}$ shows hit rates under the suppression conditions plotted as if the final item in the list was associated with $i=1$ instead of 0 , and so on. The obtained hit rates plotted in this way coincide well with the values of $H_{i}$ predicted by partial matching theory with $m=.111$. If all eight values of $i$ are considered, the intercept of the line on an imagined graph plotting the obtained hit rates as a function of the predicted hit rates is .218 , the slope is .676 , and $r=.915, d f=6, p<$ .01 ; but it is obvious that there is a primacy effect that detracts from the correlation; if only the final six triples are considered, the fit is improved; the intercept is closer to 0 (.041), the slope is closer to $1(.912)$, and $r=.971, d f=$
$4, p<.01$. Even if the activity of speaking irrelevant material aloud should add more or less than one item, the effect would only be to move the points associated with that activity that are on the solid curve of Figure 4 further up or down along the same curve.

The analyses above were concerned almost entirely with obtained hit rates, obtained FA rates, $H^{*}$, and $F A^{*}$. However, ANOVAs within each experiment were also undertaken on the $d^{\prime}, d_{L}, P_{r}, C_{L}$, and $B_{r}$ values calculated from the obtained hit and FA rates. Snodgrass and Corwin (1988) recommended calculating these values as well as hit and FA rates; $d_{L}$ and $C_{L}$ are discrimination and bias measures, respectively, from the signal detection model with the assumption that the signal and signal-andnoise distributions are logistic rather than normal; $P_{r}$ and $B_{r}$ are discrimination and bias measures, respectively, from the two-high-threshold model. These ANOVAs are available from the first author; they add nothing substantial to what has been concluded on the basis of the ANOVAs on hit rates and FA rates, and the treatment of bias when subjects are essentially guessing will be further discussed below. 


\section{DISCUSSION}

A partial matching model based on the notion that target triples coming after any given target triple that share a first digit with that triple can cause it to be forgotten by overwriting led to a prediction of $H^{*}$ rates as a function of list length for target triples with auditory components (i.e., they were heard, seen and voiced, or seen and heard). Adding two assumptions - that articulatory suppression increased the memory load and that the predicted $F A^{*}$ rate was related to the probability that a new probe shared its first digit triple with one or more of those target triples of which the first digit was still in store (i.e., had not been overwritten)--enabled us to develop a function relating $F A^{*}$ to $H^{*}$ for any list length, perceived either with clear auditory components or under articulatory suppression, that appeared to be a good predictor of actual data.

A competing family of theories, collectively known as rational response theory, failed to do this; in particular, we can state that the variable $b$ was not associated with a significant main effect for hit rates or FA rates under hearing conditions, although there was a significant main effect of $b$ for FA rates in articulatory suppression conditions only $\left[F(2,5)=4.46, M S_{\mathrm{e}}=.071, p<.01\right]$. This is reflected in the high FA rate for Burhop's experiment with $b=.67$, as can be seen, $L=7$ and 8 , as seen in Figures 2 and 4 ; indeed, in the same ANOVA the interaction of $b$ with list length was also significant $\left[F(3,316)=3.75, M S_{\mathrm{e}}=.033, p<.01\right]$.

However, we do not wish to leave the impression that partial matching theory is applicable in all cases of immediate probed recognition. As can be seen from Equations 1 and 2, rational response theory involves a guessing component that is absent from partial matching theory. If we were to find evidence obtained from immediate probed recognition tasks that are so difficult as to require such extensive guessing that the function relating obtained hit rates (on the ordinate) to obtained FA rates (on the abscissa) lay on a straight line descending through them from the point $(0,1)$, this would be prima facie evidence that rational response theory might be applicable when recognition is extremely difficult. Such data are, in fact, provided in the paper by Murray et al. (1988).

In Experiment 1 of that paper, which was carried out by Karen Smith, lists of eight digit triples (which included zero, so that $m=.10$ in these experiments) were presented at a rate of one triple every $2 \mathrm{sec}$. The lists were presented as follows: (1) The words were voiced aloud, (2) subjects spoke the word the aloud in unison with each digit triple (Condition The), or (3) subjects spelled a word aloud (Condition Spell); each word was eight letters lo: g, was spoken to the subject just before each list began, and the subject spelled each letter of the word in unison with a corresponding triple. In Experiment 2, Andrea Rowan used the same presentation rate for the eight triples. However, as each digit triple appeared on the screen, a randomly selected letter of the alphabet appeared two spaces to the right of the triple. In Condition See/Write, the subject was instructed to write down that letter (as well as trying to memorize the triple). In Condition See/Say, the subject was instructed to voice aloud the letter that alphabetically followed the letter presented with each stimulus item. Thus, if the letter $L$ was presented on the screen, the subject would say aloud $M$. In another set of conditions, the subject saw each triple at the same time as he/she heard the experimenter say a random letter that the subject had to shadow aloud (Condition Hear/Say). In Condition Hear/Write, the subject viewed the triple at the same time as hearing a random letter spoken by the experimenter (e.g., $L$ ), but wrote down the next letter of the alphabet $(M)$. In both experiments, $b$ was preset by the experimenter at .5 .

Figure 5 shows the mean obtained $(\mathrm{FA}, \mathrm{H})$ rates for the three conditions of Smith's experiment and the four conditions of Rowan's experiment plotted in the manner of Figures 2 and 4 . Clearly the points lie in a region remote from that predicted by partial matching theory with $m=$ .10 . The dotted line shows the function predicted by rational response theory with $g$ equal to .575 if Equations 1 and 2 are considered valid (below, we shall qualify this assertion); this provided a good fit to Rowan's data if the aberrant (FA,H) point for $h-w$ is ignored; Smith's data lay along a closely adjacent line predicted by rational response theory with $g=.579$. It might also be noted that the data point most removed from the partial matching curve in Figure 4, namely Burhop's (FA,H) data for $L=$ 8 , spell, $b=.67$, when added to Figure 5, also lies on the curve predicted by the rational response theory of Equations 1 and 2 with $g=.575$.

If the eight obtained FA rates shown in Figure 5 are combined with the 24 shown for the main experiment in Figure 4, the straight line relating obtained to predicted FA rates had the following values: intercept, .0034; slope, $.9782(r=.952, d f=30, p<.00001)$. That is, $90.63 \%$ of the variance in a sample of 32 obtained FA rates could be accounted for by partial matching theory given the assumption that the corresponding obtained hit rates were appropriate estimates of $H^{*}$ except when the task involved viewing eight digit triples along with a difficult articulatory suppression task, in which case rational response theory provided a better account. Note that in all the above calculations, a computer was never used to obtain estimates of "unknown" or "free" parameter values, which, when entered into an equation, would yield the predicted values to be compared with the obtained values. All the predicted values used here were calculated on the basis of known values of externally controlled variables such as list length, $b$ and $m$, and one estimated value, namely the $i$ value associated with the final target of lists viewed with articulatory suppression.

\section{EXTENSION OF PARTIAL MATCHING THEORY TO THE MIXED TEST LIST PARADIGM}

We begin this section by postulating that partial matching theory and rational response theory can be related as follows. We may remind the reader of the notation spec- 


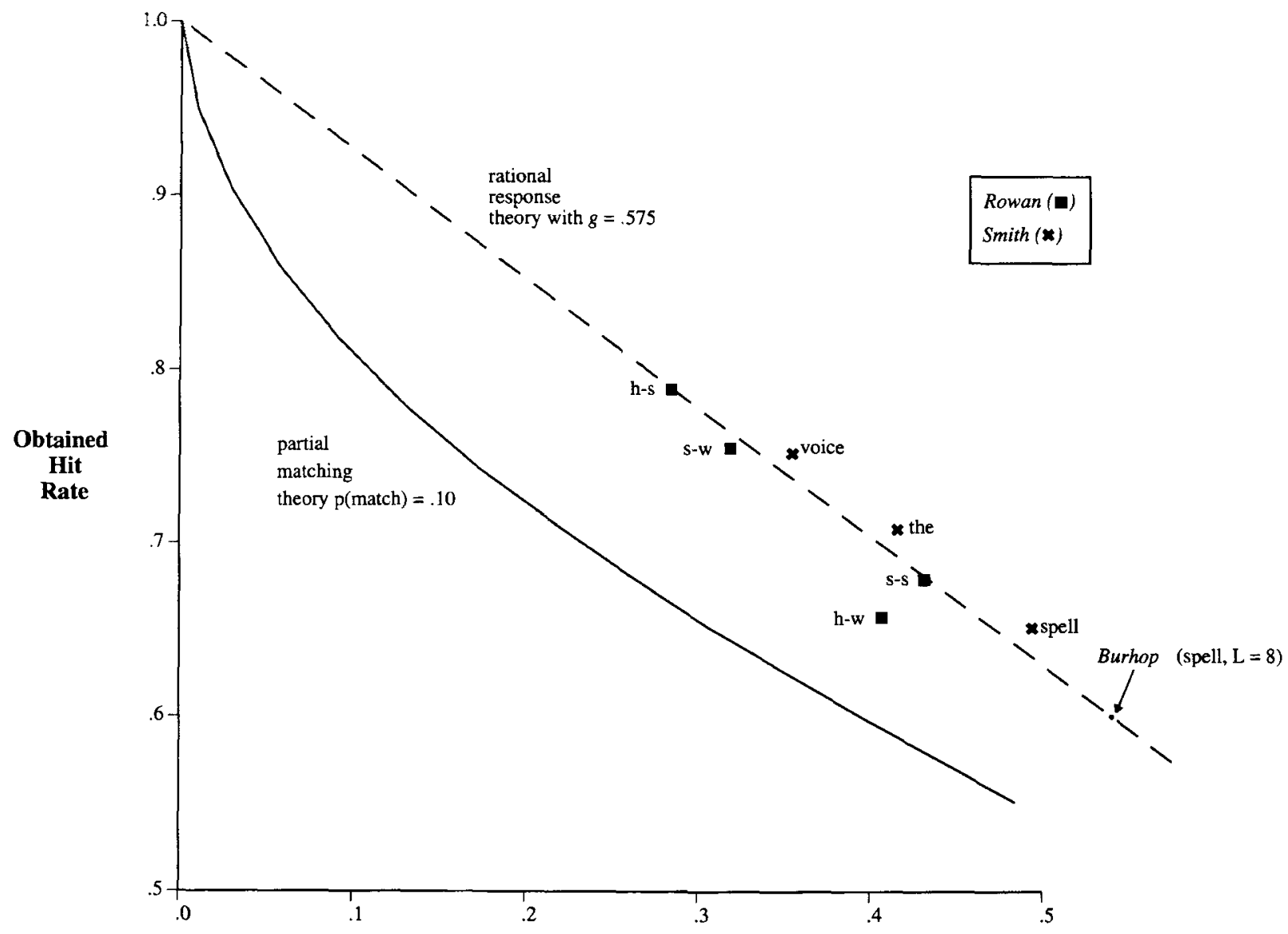

Obtained False Alarm Rate

Figure 5. Mean obtained hit rates as a function of mean obtained false alarm rates in the experiments by Smith, Rowan, and Burhop. Also shown are the curves predicted by partial matching theory with $m=.10$ and the best fitting straight line to the data of Rowan and Burhop.

ified in the introduction. A list of $L$ target triples is presumed to leave a memory representation containing $x$ triples retained in their entirety, $p$ triples retained partially (in the case of digit triples, by their first digit only), and $k$ triples forgotten because of overwriting. Partial matching theory specifies a hit rate $H^{*}$ that depends on the number $i$ of subsequent target triples according to Equation 4 and an $\mathrm{FA}$ rate $F A^{*}$ given by Equation 5 . In neither Equation 4 nor Equation 5 was there any specification concerning a guessing parameter.

According to rational response theory, the predicted hit rate $H^{\prime}$ and the predicted FA rate $F A^{\prime}$ require a guessing parameter denoted by $g$ in the introduction. Although Equations 1 and 2 specified $H^{\prime}$ and $F A^{\prime}$, respectively, it can be seen from these that

$$
H^{\prime}=(x / L)+F A^{\prime} .
$$

It was also specified that $g$ could equal $b$, the preset probability of an old probe, but not necessarily.
We are now going to suggest that Equations 1 and 2 can be modified to include $p$ in addition to $x$, and that when this is done, this modified rational response theory appears to be applicable to data collected using the mixed test list paradigm with words of high or low frequency as the target material.

The main difference between the partial matching theory and the rational response theory, as developed thus far in the context of the immediate probed recognition paradigm, is that the latter includes a guessing parameter whereas the former does not. We surmise that when a target list exceeds a critical length, retrieval can no longer be based entirely on ecphoric arousal by an old or new probe of one of the $(x+p)$ memory representations, but must involve guessing. This critical length can be surmised on commonsense grounds to be approximately equal to the memory span; alternatively, a moment's thought will indicate that when the length of the list exceeds the number of digits that can serve as first digits, 
here 9 or 10 , then if a probe has a first digit that seems familiar, it must almost certainly have indeed occurred as a partially encoded target and guesswork is required to determine whether that probe might be new. But a strictly formal approach makes use of the fact that the sum shown in Equation 4, before being divided by $L$, converges to the value $(1 / m)$. If $m$ equals, 111 , then the sum of the $H_{i}$ values as $i$ increases converges to (1/.111) - that is, 9.009. This is roughly the memory span for digits.

So we assume that once $L$ has exceeded the critical list length approximated by $(1 / m)$, guessing will be involved and that rational response theory will determine the obtained hit and FA rates. The next step is to modify rational response theory to include FAs based on partial matching as well as on unlucky guesses. We begin with the case of stimulus materials that consist of fairly well known words because then there will be little objection to the idea that, for long lists, there will be no cases where a word is genuinely overwritten in the sense of being erased forever. That is, in the mixed test list paradigm, we shall assume that $k=0$ and hence that

$$
L=x+p
$$

and therefore that

$$
(p / L)=(L / L)-(x / L)=1-(x / L) .
$$

If at the time of the recognition test all memory representations of words consist of partial representations of those words (we shall explain very shortly that what is forgotten is not an aspect of the spelling or meaning of a word, but its temporal context), then we may assume that when a new probe is presented, it may or may not arouse a feeling of strong familiarity. Let us therefore divide the memory representations of the target words into three kinds:

1. a proportion $(x / L)$ that are always responded to as "old" to old probes with probability 1 and as "new" to new probes with probability 0 if they come to mind as a new probe is presented;

2. a proportion $(n)(p / L)$ that are always responded to as "old" to new probes because, out of the proportion $(p / L)$ of partially encoded targets, a subproportion $n$ are activated because the new probe partially matches them to an extent that the subject is confident that they are genuinely old and therefore says "old" with probability 1 ;

3. a proportion $(1-n)(p / L)$ that, when a probe is presented, are not activated to the extent of arousing any feeling of familiarity, and it thus becomes a matter of guesswork as to whether the probe should be judged "old" or "new"; we surmise that when such probes appear, the subject guesses "old" with probability $b$ (corresponding to the experimentally preset probability of an old probe).

We therefore argue that, when a new probe is presented, the probability of saying "old" to it and thereby yielding an FA will be

$$
(x / L)(0)+(n)(p / L)(1)+(1-n)(p / L)(b) .
$$

In each of the three terms of this expression, the final number represents the probability of saying "old" and the preceding coefficient represents which of the above three classes of memory representation was activated by the new probe. This is the predicted FA rate according to modified rational response theory and will be labeled $F A^{* \prime}$ to indicate that it combines partial matching theory with rational response theory. Following some straightforward algebra on the above expression we find that

$$
F A^{*^{\prime}}=(n+b-n b)(p / L),
$$

and, making use of Equation 7, it follows that

$$
F A^{* \prime}=[b+n(1-b)][1-(x / L)] .
$$

Since Equation 6 specifies the predicted hit rate according to the unmodified rational response theory expressed in Equations 1 and 2, we shall argue by analogy that the predicted hit rate $H^{* \prime}$ according to modified rational response theory will also be

$$
H^{* \prime}=(x / L)+F A^{* \prime}
$$

and therefore that

$$
H^{*^{\prime}}=(x / L)+[b+n(1-b)][1-(x / L)] .
$$

Equations 8 and 9 preserve some valuable properties of Equations 1 and 2 insofar as both sets of equations make predictions about obtained hit and FA rates that are directly testable by experiment. Equations 8 and 9 make three predictions about $(\mathrm{FA}, \mathrm{H})$ data points obtained from the mixed test list paradigm. These are as follows:

1. If a set of obtained $(\mathrm{FA}, \mathrm{H})$ points are plotted on a graph labeled identically to Figures 2, 4, and 5, these points will lie on a straight line; if it is further postulated that an FA rate of 0 will necessarily be associated with a hit rate of 1 , the best fitting straight line connecting the set of obtained $(F A, H)$ points will also intersect the point $(0,1)$.

2 . Above, $n$ was defined as the probability that a new probe would partially match a partially encoded target. In the experiments with digit triples reported earlier in this paper, $n$ was identical with $m$, the probability that the first digit of a final target would be matched by the first digit of the penultimate target. However, it is not always easy to be as precise as this about the value of $n$; for example, in any experiment in which the similarity of new probes to targets is itself made an experimental variable, as was the case in the experiments conducted by Bahrick and Bahrick (1964), it may or may not be the case that $n$ can be given a precise numerical value. But the prediction can be made that if $n$ is greater in a set of Conditions A than in a set of Conditions B, other things being constant, the slope of the straight line connecting the obtained $(\mathrm{FA}, \mathrm{H})$ points of the A set to the point $(0,1)$ will be shallower than the corresponding slope connecting the points of the $B$ set.

3 . Whereas the influence of the variable $b$ on the obtained $(\mathrm{FA}, \mathrm{H})$ points in an immediate probed recognition 


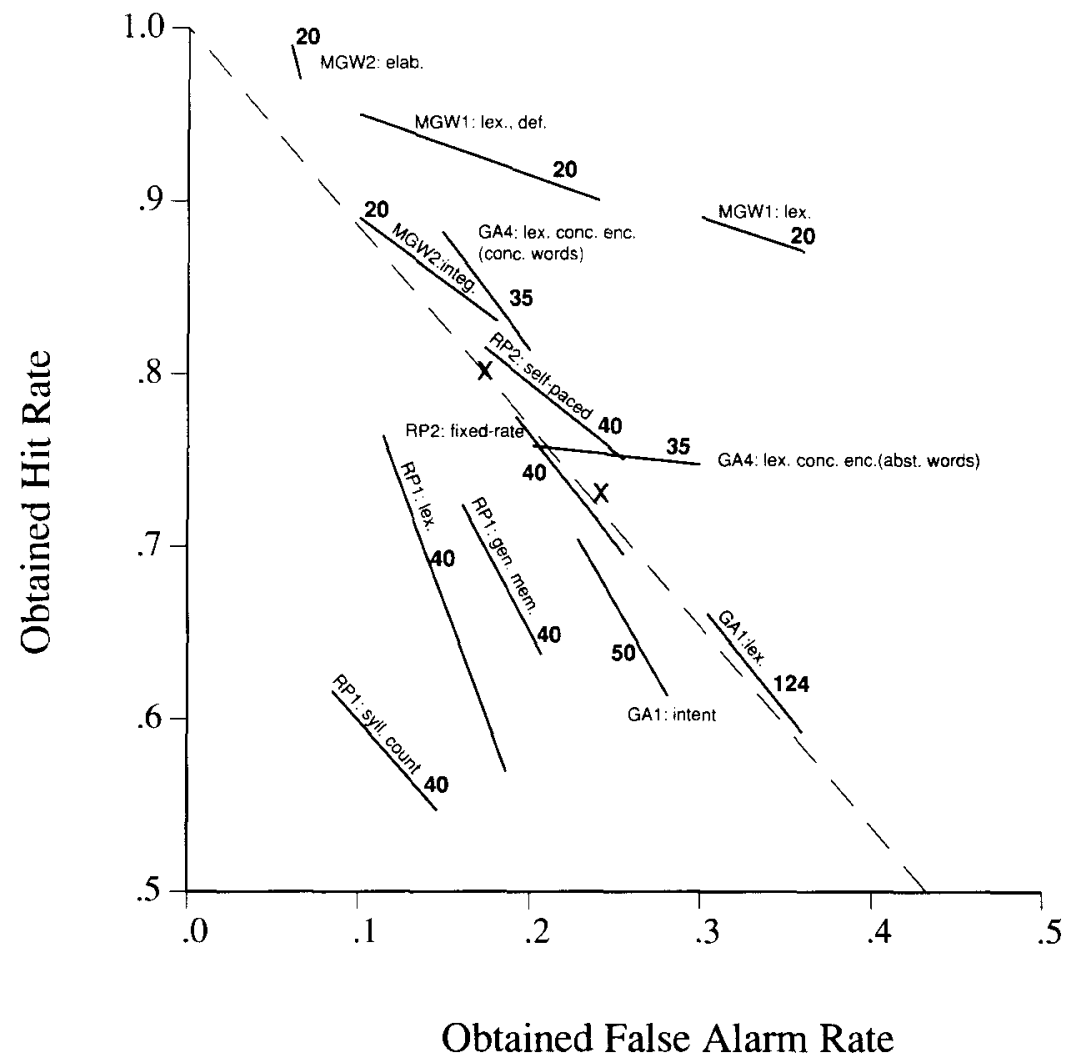

Figure 6. Obtained false alarm and corresponding hit rates from 13 experiments reported by Mandler et al. (1982), Rao and Proctor (1984), and Glanzer and Adams (1990). GA, Glanzer and Adams (1990); MGW, Mandler, Goodman, and Wilkes-Gibbs (1982); RP, Rao and Proctor (1984). Digits refer to experiment numbers. Bold numbers refer to the number of target words at each frequency. The labeling identifies the conditions in exactly the same words as were used in those papers. The leftmost point on each line represents low-frequency words; the rightmost point represents high-frequency words. The bold numbers represent the number of targets at each frequency. The dashed line is the straight line best connecting the point $(0,1)$ with the average (FA,H) point for low-frequency words (leftmost) and the average (FA,H) point for highfrequency words (rightmost); these last two points are marked with a large $X$.

task was negligible, according to Figure 2 of the present paper, Equations 8 and 9 predict that $b$, the preset probability of an old probe, will yield a significant main effect in an experiment using the mixed test list paradigm in which the probability that a lure is really old is made the focus of an experimental manipulation. We do not know of such an experiment at the time of writing.

We now ask how far Prediction 1 can be confirmed by examining previously obtained data using the mixed test list paradigm. Figure 6 takes a sample of 13 experiments drawn from three studies, those of Mandler, Goodman, and Wilkes-Gibbs (1982), Rao and Proctor (1984), and Glanzer and Adams (1990). Each single line joining 2 points (only) joins the obtained $(\mathrm{FA}, \mathrm{H})$ point for lowfrequency words at the left of the line to the obtained (FA,H) point for high-frequency words at the right of the line. The line itself is labeled with a name corresponding exactly to the name given to its condition by the experi- menters concerned; the bold number gives the number of words used for the high-frequency as well as the lowfrequency condition. This means that the list length for that line was twice the number shown. The 2 points marked with a large $\mathrm{X}$ represent the average $(\mathrm{FA}, \mathrm{H})$ points for all 13 experiments for low-frequency words (the left $X$ ) and for high-frequency words (the right $\mathrm{X}$ ).

These 2 points lie on a line whose slope deviates only slightly from -1 (the dashed line in Figure 6). For lowfrequency words, the FA and $H$ values were .1665 and .8016 , respectively; for high-frequency words, they were .2334 and .7333 , respectively. The straight line best fitting those 2 points plus the point $(0,1)$ had an intercept with the ordinate of .9985 , a slope, $S_{f}$, of -1.152 , and an $r$ value of -.9995 ; the estimated value of $g$ was 4648 , which just misses the value of .5 , which might be expected in a general sense if we are averaging over many experiments but in a more particular sense if the value .5 is a 
direct reflection of the fact that $b$ equaled .5 in all of these studies. We might here have evidence consistent with Prediction 3 above.

However, it is obvious from Figure 6 that Equations 1 and 2 are adequate only for the two average $(\mathrm{FA}, \mathrm{H})$ values shown by the two Xs. Many of the obtained $(\mathrm{FA}, \mathrm{H})$ points are associated with mirror effects that have slopes visibly different from -1 . But an explanation of the pattern of mirror effects shown in Figure 6 can be offered, as follows. Most of the mirror effects with slopes steeper than -1 were carried out by Rao and Proctor (1984), using lists of about 40 low- and 40 high-frequency words presented at a rate such that we can calculate the average retention interval to have been $8-10 \mathrm{~min}$ for any single word with respect to the presentation of its corresponding old probe. Most of the mirror effects with slopes flatter than -1 were carried out by Mandler et al. (1982), who showed the first probe word either $5 \mathrm{~min}$ or $24 \mathrm{~h}$ after the last target word. These authors also showed that the FA rate was much greater after $24 \mathrm{~h}$ than it was after $5 \mathrm{~min}$ for identical target words. In a moment we shall try to account for these findings; but we first have to define what is meant by $p$, the number of partially encoded memory representations of words available at the time of the probe. It is easy to understand what is meant by a partially encoded digit triple; but how do you partially encode a word?

We shall surmise that all target words are encoded not only in terms of meaning but also in terms of the context of a particular target list. If, in a probe list, a probe word is presented that seems familiar to the subject, but he/she is uncertain about whether the word is familiar because it was in the target list or merely because he/she has seen it before in some other context (another list, or everyday experience), then some FAs are likely to arise; this situation can be called a failure of list differentiation. Postman and Underwood (1973) used this phrase when discussing the various sources of retroactive inhibition that could reduce performance in serial learning and pairedassociates learning. In particular, it would be difficult to ascribe high-frequency words to the target list with certainty since they often also occur in everyday life; similarly, FAs could result if a target list contained several words from one semantic category and a new probe word was also a word within that category (Roediger \& McDermott, 1995). In the mixed test list paradigm, the equivalent of a digit triple coded by its first digit only might be a target word encoded without its embedding list context. Any new probe that shared a semantic context with that target word, but did not match it in terms of list context, might be responded to as "old" on the grounds that it "partially matched" a stored word in terms of its meaning, even though the list context of the word was not available at the time of that probe.

Now let us assume that the passing of time also involves some forgetting of target context, though not of target meaning. At a short retention interval, an unfamiliar probe that happens to be new will be fairly easy to recognize as new because it will be clearly distinct from those memories of target words still retained along with their context. But at long retention intervals, when target contexts are more likely to have been forgotten, unfamiliar probes will be harder to recognize as new. There will be a stronger tendency to mistakenly say "old" to any probe that seems familiar from everyday contexts because the familiarity is wrongly attributed to the probe's having occurred in the context of the target list.

This tendency will be reflected in a value of $g$ that exceeds $b$ (here, .5). In turn, this will be reflected in a slope $S_{f}$ that lies between -1 and 0 on graphs labeled as in Figure 6 (such a line will appear visually flatter than the diagonal of Figure 6). If the slope $S_{f}$ lies between -1 and 0 , it is shown in the Appendix that $g$ will exceed .5; if, as is specified by Equations 8 and 9, we can set $g$ equal to $[b+n(1-b)]$, and $b=.5$, the term $n(1-b)$ will be positive and subjects will respond "old" on more than half of the trials involving unfamiliar probes; in such cases the FA rate will be higher than expected on the basis of unlucky guessing only, presumably reflecting matches between the meanings of new probes (encoded in the context of the test list) and the meanings of memory representations of target words. But these matches are only "partial" because the context of these memory representations (the study list) has been forgotten. If the slope lies between -1 and $\infty$ (appearing visually steeper than the diagonal of Figure 6), $g$ will be less than .5 , and if $b=.5$, the term $n$ $(1-b)$ will be negative. This can be interpreted to mean that subjects responded "new" on more than half the trials involving unfamiliar probes. In such cases, even though $n$ might exceed 0 , there will be a systematic tendency to have an FA rate lower than expected on the basis of guessing only. These remarks are consistent with the explanation of the pattern shown in Figure 6 as being due to two facts. The data collected by Rao and Proctor (1984) reflected a low incidence of FAs because new probes were clearly identified as "new" since the retention interval was only a few minutes. But the data collected by Mandler et al. (1982) had a high incidence of FAs because new probes were often mistakenly judged to be "old" because the long retention interval used by Mandler et al. allowed the context of the target words to have been forgotten.

It may also be noted that the experiment that yielded (FA,H) data most consistent with a guessing strategy in which $g=b=.5$ was that of Experiment 1 of Glanzer and Adams (1990), who used exceptionally long lists and incidental learning of the target words. When the list was reduced from 124 to 50 words of each frequency, and learning was intentional, the ability to recognize new probes as new was enhanced, a fact reflected in a curve (GAl:intent) with a slope associated with a value of $g$ less than .5 .

Some other practical issues connected with the estimation and interpretation of $g$ and $n$, given two or more obtained $(\mathrm{FA}, \mathrm{H})$ points, are also discussed in the Appendix. A similar line of argument could apply to short target lists of digit triples if the presence of conditions 
such as articulatory suppression also implied that guesswork was operative. So, for example, the data of Figure 5, in which $g$ for Rowan and Burhop's data was estimated to be .575 and $g$ for Smith's data was estimated to be .579 , are compatible with the notion that $g=[b+n(1-b)]$ with $b=.5$ and $n=.15$ for Rowan and Burhop's data and $b=.5$ and $n=.158$ for Smith's data.

\section{Relation of the Present Work to the Earlier Literature on Mirror Effects}

It is essential to be more specific about how the above line of reasoning relates to the achievements of Glanzer et al. (1993), who have been able to show how many experiments using the mixed test list paradigm, including some of those shown in Figure 6, yielded mirror effects that could be well accounted for by using the signal detection model. For example, these authors were able to provide straight lines relating obtained data to predicted data that were associated in some cases with an $r$ value of .98 (see Figure 7 of their paper). We do not have any evidence that their model, or the signal detection model of Shiffrin and Steyvers (1997), can actually be validly criticized on psychological or quantitative grounds, but we do wish to assert that the evidence offered in the present paper suffices for us to claim that distribution-free models incorporating partial matching can offer an alternative approach that cannot, at present, be rejected out of hand. In particular, we claim that the distribution-free approach offers two features at present missing in the literature on mirror effects in the context of signal detection theory.

First, we have tried to provide a theory in which the values of the important variables can be controlled in advance of an experiment; there are no references to what Murray (1987) has called "internal scalable magnitudes." Such magnitudes include sensation strength, habit strength, memory strength, and attention strength. However, the theory of Glanzer et al. (1993) has as its core the notion that a subject pays attention to a given number of features in a target and in an associated probe. Since this number is hard to measure, the success of their model depends on how well their theory of the mirror effect can account for their obtained data. Such an evaluation involves finding the unknown values of certain variables that, when inserted into the appropriate equations, yield imagined data that deviate minimally from the obtained data. In the case of yes-no recognition data, the matching of obtained data to the imagined data can best be achieved by using confidence ratings to estimate slopes of receiver operating curves that in turn are used to estimate the variances of the four distributions (high- and low-frequency old and new probes), from which, using a procedure too complicated to summarize here (see p. 555 of their article), they estimated imagined values of several variables using a computer. These values were such that the predicted hit and FA rates predicted on the basis of these values deviated minimally from obtained hit and FA rates.
They were forced to use this complicated procedure because if, from any experiment, the experimenter has available two obtained hit rates (for high- and low-frequency words) and two corresponding FA rates, these four rates by themselves are insufficient for quantitative fitting of the model to those data because more than four parameters are necessary when quantitative characteristics of the distributions in the model also have to be specified. (The situation is rather like having two equations each with three unknown values; the equations are insoluble by analytic methods. Although Equations 8 and 9 can be solved for $x$ and $n$, if we know $b$ and obtained FA and hit rates, in practice situations can arise where $b$ is not known in advance; hence this problem can also arise in the context of modified rational response theory.)

On the other hand, it can be argued that, since Glanzer et al. (1993) have accounted in their model for confidence ratings as well as for hit and FA rates, they have achieved more than we have in the sense of accounting for more varieties of data. Moreover, they were able to account for more of the variance using their model $(98 \%$ in some cases) than we were with ours $(90.63 \%$ in the case of the data shown in Figures 4 and 5). However, goodness-of-fit tests, though usually persuasive, do not always help us in deciding between theories. Van Zandt and Ratcliff (1995) showed, for example, that curve fitting could not reasonably be used in practice to decide between the merits of the hypothesis that reaction times are distributed according to a gamma distribution and those of the hypothesis that the distribution is a convolution of the exponential and the Gaussian. Indeed, if goodness-of-fit tests indicate no compelling differences between competing models as predictors of data, perhaps those competing models are not really "competing" but are alternative expressions of some underlying regularity. For instance, Laming and Laming (1996) have argued that Murray's (1993) evidence showing that both Fechner's logarithmic function and Stevens's power function were equally good predictors of some data on lightness scaling collected in Fechner's time by Delboeuf is actually consistent with $\mathrm{D}$. Laming's own view that these two functions are different aspects of a third, underlying, process (to do with contrast detection).

Second, the distribution-free theories discussed in the present paper are able to make predictions about exact magnitudes of the hit rates and corresponding FA rates to be expected as a consequence. So far as we are aware, no models of recognition that are in the tradition of signal detection theory predict magnitudes of this kind in advance of experiments, although, of course, many wellknown models of recognition, such as those reviewed by Clark and Gronlund (1996), attempt to give retrospective accounts of previously obtained data, accounts that are supported by demonstrations that these obtained data can be accounted for by the models provided that the appropriate values of each of the variables in the model are inserted into the equations. There are no cases in the pres- 
ent paper of this kind; spreadsheets were used extensively for carrying out arithmetic, but no search program such as Stepit, Linreg, or Praxis has yet had to be used in developing our theories. In particular, we have been unable to extract from the literature a graph with the axes labeled in a manner corresponding to those of Figures 2, 4,5 , and 6 but in which signal detection theory is used to predict exact FA rates given hit rates that were themselves predicted on the basis of a knowledge of the values of one or more experimentally controlled variables.

We must, however, also note that both the distributionfree and distribution-centered approaches share key features. Both assert that hit rates are a function of the degree to which old probes and their corresponding memory representations share features, and both assert that FA rates can rise when new probes accidentally share features with memory representations of old targets. Both approaches make use of internal feelings of familiarity as guidelines on which to base decisions as to whether to respond "old" or "new" to a probe, but both partial matching theory and modified rational response theory lay more emphasis on the difference between partially and wholly encoded memory representations than does signal detection theory. Because of this, these distribution-free theories are in the Gestalt tradition (Murray, 1995, Chaps. 3 and 4). On the other hand, both distribution-free theories share with the model of Shiffrin and Steyvers (1997) the notion that stored memory representations may be partial or "incomplete," to use the word those authors apply to their "images."

Moreover, both approaches can represent response biases numerically when the memory representations are so poor as to induce guessing; the signal detection model shows how hit and FA rates both rise as criterion settings become increasingly liberal, given a constant value of $d^{\prime}$, whereas the modified rational response theory shows how hit rates and FA rates both rise if the term $(n)(p / L)$ is greater in value in one experimental condition than in another, other things being equal. Neither the $d^{\prime}$ (or $d_{L}$ ) of signal detection theory nor the $(x / L)$ of rational response theory is identical to the obtained hit rate; both represent measures of discrimination based on ecphoric memory activation. An obtained "hit rate" usually includes some lucky guesses, denoted indirectly in signal detection theory by $\beta$ or $C$, and denoted in the modified rational response theory by $[b+n(1-b)][1-(x / L)]$. More generally, rational response theory as reflected in Equations 1 and 2 is consistent with two-high-threshold theory; modified rational response theory as reflected in Equations 8 and 9 represents a step in the direction of an analogy to a shifting criterion setting insofar as the value of $(n)(p / L)$ is not fixed but can also shift. If so, it is possible that the distribution-free and distribution-based approaches, both of which can be demonstrated to yield predicted values that are a good "fit" to obtained values, actually represent different approaches to a third, underlying, variable to do with the "degree of wholeness" of individual memory representations.
Yet perhaps the most important suggestion made in the present paper is contained in the notion that, for list lengths below $(1 / \mathrm{m})$, partial matching theory provides a good description of expected hit and FA rates, whereas for list lengths above that level, a rational response theory modified to include partial matching between new probes and partial memory representations, as well as guessing, can apparently provide a starting point for further investigations. It will be imperative in such investigations to be more precise about two questions unresolved in the present paper. As noted in the previous paragraph, we need to know more about the mathematical relationships between distribution-free theories and theories that do involve assumptions about distributions. For example, Figures 2, 4, 5, and 6 of the present paper show individual data points of the form $(\mathrm{FA}, \mathrm{H})$ that in terms of the signal detection model can be taken to represent FA and hit rates obtained with a single (here, undetermined and undiscussed) criterion setting; that is, lines connecting a series of such (FA,H) points might represent isobias functions. And, as noted in the present paragraph, we need to know more about the critical list length for any given kind of target material - the list length below which partial matching theory appears operative and above which a version of rational response theory appears operative. It is hoped that these two questions, and others, will be discussed more fully in future contributions.

\section{REFERENCES}

BAhrick, H. P., \& BAHRICK, P. O. (1964). A re-examination of the interrelations among measures of retention. Quarterly Journal of Experimental Psychology, 16, 318-324.

Brown, J., Lewis, V. J., \& Monk, A. F. (1977). Memorability, word frequency, and negative recognition. Quarterly Journal of Experimental Psychology, 29, 461-473.

Clark, S. E., \& Gronlund, S. D. (1996). Global matching models of recognition memory: How the models match the data. Psychonomic Bulletin \& Review, 3, 37-60.

Elliott, P. B. (1964). Tables of $d^{\prime}$. In J. A. Swets (Ed.), Signal detection and recognition by human observers (pp. 651-84). New York: Wiley.

Glanzer, M., \& Adams, J. K. (1985). The mirror effect in recognition memory. Memory \& Cognition, 13, 8-20.

GlanZER, M., \& ADAMS, J. K. (1990). The mirror effect in recognition memory: Data and theory. Journal of Experimental Psychology. Learning, Memory, \& Cognition, 16, 5-16.

Glanzer, M., Adams, J. K., Iverson, G. J., \& Kim, K. (1993). The regularities of recognition memory. Psychological Review, 100, 546-567.

GreEnE, R. L. (1996). Mirror effect in order and associative information: Role of response strategies. Journal of Experimental Psychology: Learning, Memory, \& Cognition, 22, 687-695.

HeIT, E., \& Jones, C. M. (1997, March). Two-process models of recognition memory: Recall-to-accept or recall-to-reject? Paper presented at a meeting of the Experimental Psychology Society, Oxford.

Hintzman, D. L., Caulton, D. A., \& Curran, T. (1994). Retrieval constraints and the mirror effect. Journal of Experimental Psvchology: Learning, Memory, \& Cognition, 20, 275-289.

Hockley, W. E. (1994). Reflections of the mirror effect for item and associative recognition. Memory \& Cognition, 22, 713-722.

KuČera, H., \& Francis, W. N. (1967). Computational analysis of present-day American English. Providence: Brown University Press. LAming, J., \& LAming, D. (1996). J. Plateau: On the measurement of 
physical sensations and on the law which links the intensity of these sensations to the intensity of the source. J. Plateau: Report on "Psychological study: Theoretical and experimental research on the measurement of sensations, particularly sensations of light and fatigue" by Mr. Delboeuf. Psychological Research, 59, 134-144.

Logie, R. H., Della Sala, S., Lajacona, M., Chalmers, P., \& WYNN, V. (1996). Group aggregates and individual reliability: The case of verbal short-term memory. Memory \& Cognition, 24, 305-321.

Mandler, G., Goodman, G. O., \& Wil Kes-GrbBs, D. L. (1982). The word-frequency paradox in recognition. Memory \& Cognition, 10 , 33-42.

MCCormack, P. D., \& SWENSON, A. L. (1972). Recognition memory for common and rare words. Journal of Experimental Psychology, $95,72-77$.

MURRAY, D. J. (1987). A perspective for viewing the integration of probability theory into psychology. In L. Krüger, G. Gigerenzer, \& M. S Morgan (Eds.), The probabilistic revolution: Vol. 2. Ideas in the sciences (pp. 73-100). Cambridge, MA: MIT Press.

MurRay, D. J. (1993). A perspective for viewing the history of psychophysics. Behavioral \& Brain Sciences, 16, 115-186.

MURRAY, D. J. (1995). Gestalt psychology and the cognitive revolution. London: Prentice-Hall, Harvester Wheatsheaf.

Murray, D. J., Birch, D. E., Chase, R. S., Eyolfson, B. H., \& Simms, K. A. M. (1991). The effect of vocalization at presentation on shortterm recognition of three-digit numbers. Canadian Journal of Psychology, 45, 462-473.

Murray, D. J., Boudreau, N., Burggraf, K. K., Dobell, L., Guger, S. L., Leask, A., Stanford, L., Tate, T. L., \& Wheeler, M. (in press). A grouping interpretation of the modality effect in immediate probed recognition. Memory \& Cognition.

Murray, D. J., Rowan, A. J., \& Smith, K. H. (1988). The effect of articulatory suppression on short-term recognition. Canadian Journal of Psychology, 42, 424-436.

NeATH, I., \& NaIRNE, I. S. (1995). Word-length effects in immediate memory: Overwriting trace decay theory. Psychonomic Bulletin \& Review, 2, 429-441.

Postman, L., \& Underwood, B. J. (1973). Critical issues in interference theory. Memory \& Cognition, 1, 19-40.

RaO, K. V., \& Proctor, R. W. (1984). Study-phase processing and the word frequency effect in recognition memory. Journal of Experimental Psychology: Learning, Memory, \& Cognition, 10, 386-394.

Roediger, H. L., \& MCDermotT, K. B. (1995). Creating faise memories: Remembering words not presented in lists. Journal of Experimental Psychology: Learning, Memory, \& Cognition, 21, 803-814.

ShiffRIN, R. M., \& SteYVERS, M. (1997). A model for recognition memory: REM - Retrieving effectively from memory. Psychonomic Bulletin \& Review, 4, 145-166.

SNodgrass, J. G., \& Corwin, J. (1988). Pragmatics of measuring recognition memory: Applications to dementia and amnesia. Jourmal of Experimental Psychology: General, 117, 34-50.

VAN ZANDT, T., \& RATCLIFF, R. (1995). Statistical mimicking of reaction time data: Single-process models, parameter variability, and mixtures. Psychonomic Bulletin \& Review, 2, 20-54.

VOKEY, J. R., \& READ, J. D. (1992). Familiarity, memorability, and the effect of typicality on the recognition of faces. Memory \& Cognition, 20, 291-302.

WiCKelgren, W. A., \& Norman, D. A. (1966). Strength models and serial position in short-term memory. Journal of Mathematical Psychology, 3, 316-347.

\section{APPENDIX}

To avoid confusion, we shall use the letter $h$ to denote "hit rate" in a generic sense, $f$ to denote "false alarm rate" in a generic sense, $a$ to denote "proportion of hits due to ecphoric arousal" (as opposed to lucky hits), and $g$, as before, to denote the probability of saying "old" to an unfamiliar probe.

Rational response theory involves equations of the generic form:

$$
\begin{aligned}
& h=a+g(1-a)=a+f \\
& f=g(1-a) .
\end{aligned}
$$

When $f$ is plotted on the abscissa and $h$ on the ordinate, the slope $S_{f}$ of the straight line describing $h$ as a function of $f$, if rational response theory holds, will be negative.

If two conditions (e.g., conditions involving high- as opposed to low-frequency words) yield hit rates $h_{1}$ and $h_{2}$ with corresponding false alarm (FA) rates $f_{1}$ and $f_{2}$ and corresponding $a$ values of $a_{1}$ and $a_{2}$, the slope $S_{f}$ is given by

$$
\begin{aligned}
S_{f} & =\left(h_{1}-h_{2}\right) /\left(f_{1}-f_{2}\right) \\
f_{1}-f_{2} & =g\left(1-a_{1}\right)-\left[g\left(1-a_{2}\right)\right] \\
& =g\left(-a_{1}+a_{2}\right) \\
h_{1}-h_{2} & =\left(a_{1}+f_{1}\right)-\left(a_{2}+f_{2}\right) \\
& =(-1)\left(-a_{1}+a_{2}\right)+g\left(-a_{1}+a_{2}\right)
\end{aligned}
$$

Substituting these expressions for $\left(h_{1}-h_{2}\right)$ and $\left(f_{1}-f_{2}\right)$ into Equation $\mathrm{A} I$ and dividing throughout by $\left(-a_{1}+a_{2}\right)$ yields

$$
S_{f}=(-1+g) / g \text {, }
$$

from which it follows that

$$
g=(-1) /\left(S_{f}-1\right) \text {. }
$$

If $S_{f}=-1$, then from Equation $\mathrm{A} 2$ it follows that

$$
g=(-1) /(-1-1)=(1 / 2)=.5 \text {. }
$$

To estimate $n$ as given in Equations 8 and 9 of the text, we need only calculate $n=(g-b) /(1-b)$. Given that Equations 8 and 9 are equivalent to Equations 1 and 2 with $g$ in the latter two equations replaced by $[b+n(1-b)]$, the value of the term $n(1-b)$ will be positive when $g>b$, zero when $g=b$, and negative when $g<b$.

If $h$ is plotted on the abscissa and $f$ on the ordinate, the slope $S_{h}$ of the straight line describing $f$ as a function of $h$ will not equal $S_{f}$; it will equal $1 / S_{f}$. Consequently $S_{h}$ will equal $g /(-1+g)$. The equation for $g$ will then be

$$
g=S_{h} /\left(S_{h}-1\right) \text {. }
$$

But for any set of $K$ experimentally obtained hit and FA rates, $g$ will be the same whether calculated from $S_{f}$ or from $S_{h}$. So will $r$, a measure of how well the straight line fits the $K$ data points. $K$ can be increased to $(K+1)$ data points if we add the point $(0,1)$ for $S_{f}$ or $(1,0)$ for $S_{h}$.

Since the number of degrees of freedom associated with $r$ is $(K-2)$, very high values of $r$ are necessary if the data from typical experiments on the mirror effect are to be used as a basis for estimating actual values of $a$. Moreover, sometimes it happens that a given pair of $(\mathrm{FA}, \mathrm{H})$ points quite clearly cannot be connected by a straight line to the point $(0,1)$; for example, in Figure 6 the mirror effect obtained in the condition GA4:lex. conc. enc.(abst words) stands out as different from the others in this respect. There is nothing in the format of Equations 8 and 9 of the modified rational response theory that would make this problematic; there is no necessity to believe that, if the obtained FA rate were 0 , the associated hit rate would have to be 1 . Hence there is nothing to prevent us from affirming that an FA rate of 0 could be associated with a hit rate less than 1 if for some reason the experimental conditions were such as to encourage missed signals

(Manuscript received December 23, 1996; revision accepted for publication August 13, 1997.) 